\title{
Thevenin Equivalent Method for Dynamic Contingency Assessment
}

\author{
Møller, Jakob Glarbo; Jóhannsson, Hjörtur; Østergaard, Jacob
}

Published in:

Proceedings of IEEE Power \& Energy Society's General Meeting 2015

Link to article, DOI:

10.1109/PESGM.2015.7286120

Publication date:

2015

Document Version

Peer reviewed version

Link back to DTU Orbit

Citation (APA):

Møller, J. G., Jóhannsson, H., \& Østergaard, J. (2015). Thevenin Equivalent Method for Dynamic Contingency Assessment. In Proceedings of IEEE Power \& Energy Society's General Meeting 2015 IEEE. https://doi.org/10.1109/PESGM.2015.7286120

\section{General rights}

Copyright and moral rights for the publications made accessible in the public portal are retained by the authors and/or other copyright owners and it is a condition of accessing publications that users recognise and abide by the legal requirements associated with these rights.

- Users may download and print one copy of any publication from the public portal for the purpose of private study or research.

- You may not further distribute the material or use it for any profit-making activity or commercial gain

- You may freely distribute the URL identifying the publication in the public portal

If you believe that this document breaches copyright please contact us providing details, and we will remove access to the work immediately and investigate your claim. 


\section{Thevenin Equivalent Method for Dynamic Contingency Assessment}

\author{
Jakob Glarbo Møller \\ jglmo@elektro.dtu.dk
}

\author{
Hjörtur Jóhannsson \\ hjjo@elektro.dtu.dk
}

\author{
Jacob Østergaard \\ joe@elektro.dtu.dk
}

\begin{abstract}
A method that exploits Thevenin equivalent representation for obtaining post-contingency steady-state nodal voltages is integrated with a method of detecting post-contingency aperiodic small-signal instability. The task of integrating stability assessment with contingency assessment is challenged by the cases of unstable post-contingency conditions. For unstable postcontingency conditions there exists no credible steady-state which can be used for basis of a stability assessment. This paper demonstrates how Thevenin Equivalent methods can be applied in algebraic representation of such bifurcation points which may be used in assessment of post-contingency aperiodic small-signal stability. The assessment method is introduced with a numeric example.
\end{abstract}

Index Terms-Power system analysis computing, Power system stability, Thevenin equivalent, Algorithms

\section{INTRODUCTION}

A power system solver based on Thevenin equivalent representations was previously demonstrated as a mean of static security assessment for the purpose of obtaining postcontingency steady-state nodal voltages [1]. This paper develops the Thevenin equivalent static contingency assessment (TESCA) with the purpose of evaluating aperiodic small signal stability following a contingency.

The contingency assessment is greatly important for the operational security of power systems. The purpose is to adapt system operation to mitigate the severity of faults likely to occur. Dynamic contingency assessment should apply intelligent methods in evaluating system security such that the distance to instability is assessed with great confidence. This involves expanding the evaluation of operational limits to include not just static flow and voltage constraints but also those limitations to system stability that cannot directly be represented by a static flow or voltage limit. Dynamic limits to stable operation interferes with convergence of power-flow methods. This was identified by Thorp et al. for NewtonRaphson's method when applied to cases of voltage instability [2], [3].

Parameter continuation methods such as continuation power flow can be designed with good convergence properties at and around the bifurcation points. Markarov et al. have formulated a method for defining a multidimensional wide-area security

Authors are with Center for Electric Power and Energy at Department for Electrical Engineering, Technical University of Denmark.

The SOSPO project is funded by Danish Council for Strategic Research under grant agrreement no. 11-116794 region through off-line simulations [4]. This approach combines parameter continuation with contingency assessment in approximation of boundaries to a wide-area security region. The boundaries are used in on-line security assessment where the security margin is obtained as the distance from a current operating point to the nearest boundary.

Off-line estimates of a security region are useful for operation near a planned operating point. With more degrees of freedom induced by integrating large shares of intermittent generation real-time assessment of the security boundaries and margins become necessary. The vast computational burden of parameter continuation methods makes it undesirable to apply them to real-time operation.

Representing power systems by Thevenin equivalents have allowed algebraic formulation of bifurcation points. It was used by Dunlop et al. which in 1979 expressed the loadability of transmission lines in terms of surge impedance load as a function of line length [5]. Thevenin equivalent representation was also used by Jóhannsson et al. in developing a method for assessing and visualising distance to aperiodic small-signal instability [6], [7]. This research was continued in a project on secure operation of sustainable power systems (SOSPO) [8]. With special focus on real-time assessment several project contributors have found that Thevenin equivalent methods are efficient and give credible representation of system performance when used for assessment of various phenomena in power system stability. Weckesser et al. studies methods for assessing transient stability in real-time and finds that Thevenin equivalent methods are suitable alternatives [9]. Perez et al. conducted a study of methods for assessing distance to voltage collapse and Thevenin equivalent methods were found computationally efficient [10]. In a recent publication improvements to the definition of distances to voltage collapse was made to include over-excitation limitations [11]. Sensitivites derived on basis of Thevenin equivalent representation have been applied in detection of transient voltage sags caused by rotor swings in large generating units[12]. Other Thevenin equivalent based sensitivities have been derived for the purpose of managing controllable loads as remedial action to small-signal instability [13].

A fundamental necessity for applying thevenin equivalent methods in assessing post-contingency distance to instability is that a post-contingency steady-state exist. This criteria has been investigated in the following. The next sections present the formulation of the Thevenin equivalent method for 
contingency assessments. Hereafter the method for detecting aperiodic small-signal instability in contingency assessment will be introduced and tested.

\section{Method OF CONTINGEnCY ASSESSMENT}

Methods for solving power flows rely on boundary values. Similar to Newton-Raphson's power flow method the Thevenin equivalent method assumes voltage magnitudes at certain nodes to be known. The majority of power injection is situated at these nodes because the generation system commonly integrates voltage control. There is hence a distinction between voltage controlled nodes and non-controlled nodes. Any load connected to the non-controlled nodes are in the Thevenin equivalent method modelled by a constant impedance.

A generating unit is connected to a voltage controlled node $i$ while the remaining power system can be represented by a Thevenin equivalent. This forms a two-source equivalent from which certain relations can be derived analytically.

The two-source equivalent allow formulation of an expression for a $P-\delta$ curve from which the voltage angle at node $i$ may be determined on basis of the active power injection and the Thevenin equivalent.

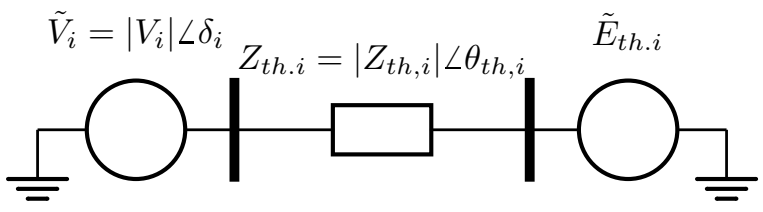

Fig. 1. Two-source equivalent seen from node $i$

$$
\delta_{i}=\arccos \left(\frac{P_{i}\left|Z_{t h, i}\right|}{\left|V_{i}\right| \cdot\left|E_{t h, i}\right|}-\frac{R_{t h, i}\left|V_{i}\right|}{\left|Z_{t h, i}\right| \cdot\left|E_{t h, i}\right|}\right)+\theta_{t h, i}
$$

Knowledge to active power injection $\left(P_{i}\right)$ and the Thevenin equivalent $\left(Z_{t h, i}\right.$ and $\left.\tilde{E}_{t h, i}\right)$ following a contingency allow determination of the post-contingency nodal voltage at bus $i$. The loss-of-line contingency is suitable for a simple proof of concept. Loosing a transmission line will usually not shift dispatched power from one unit to the other. Hence, the postcontingency power injection is roughly equivalent to the precontingency power injection for loss-of-line contingencies.

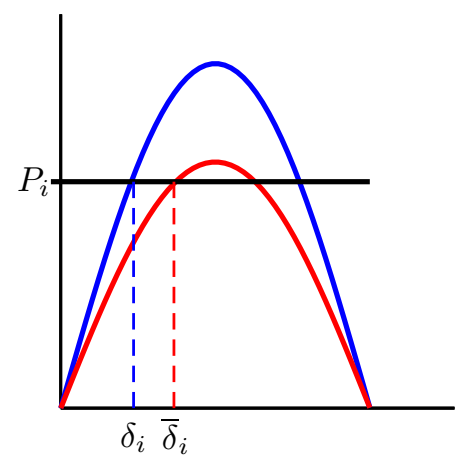

Fig. 2. Examples of pre- and post-contingency $P-\delta$ curves
Thus, when the post contingency Thevenin equivalent can be obtained it is also possible to obtain the post-contingency steady-state nodal voltages by solving for the voltage angles while assuming constant voltage magnitudes at voltage controlled nodes.

\section{A. Obtaining Thevenin equivalents}

The Thevenin equivalents can be obtained from an initial system representation given by the nodal equations;

$$
\left[\begin{array}{c}
0 \\
I_{v c}
\end{array}\right]=\left[\begin{array}{cc}
\mathbf{Y}_{n c} & \mathbf{Y}_{l i n k} \\
\mathbf{Y}_{l i n k}^{T} & \mathbf{Y}_{v c}
\end{array}\right]\left[\begin{array}{c}
V_{n c} \\
V_{v c}
\end{array}\right]
$$

where subscripts $v c$ and $n c$ indicate nodes with and without automatic voltage control respectively. The upper left block matrix $Y_{n c}$ is the admittance matrix on all non-controlled nodes. The diagonal of $Y_{n c}$ is modified according to (2) to represent all loads by their admittance value. By it-self $\mathbf{Y}_{n c}$ represents a system where all voltage sources have been short-circuited. The Thevenin impedance seen from a voltage controlled node $i$ can be obtained by concatenating the block admittance matrix $\mathbf{Y}_{n c}$ with the admittance elements corresponding to node $i$. The Thevenin impedance seen from node $i$ is equal to the open circuit voltage when injecting a unit current in node $i$.

$$
Z_{t h, i}=\left.v_{i}\right|_{I_{i}=1} ^{O C}
$$

The Thevenin impedance is therefore a solution to the inverse problem stated in (4).

$$
\left[\begin{array}{c|c}
\mathbf{Y}_{\mathbf{n c}} & Y_{l i n k,(\bullet, i)} \\
\hline Y_{l i n k,(i, \bullet)} & Y_{i i}
\end{array}\right]\left[\begin{array}{c}
\vdots \\
\hline v_{\left.i\right|_{I_{i}=1} ^{O C}}
\end{array}\right]=\left[\begin{array}{c}
\mathbf{0} \\
\hline 1
\end{array}\right]
$$

The Thevenin equivalent voltage is a sum of contributions by every voltage source in the system. Each contribution is scaled and rotated by a complex coefficient, which was named grid transformation coefficient $(G T C)$ in [14].

$$
\tilde{E}_{t h_{i}}=\sum_{j \neq i}^{k} G T C_{(i, j)} \cdot \tilde{V}_{j}
$$

The grid transformation coefficient with which the voltage at a node $j$ participates in the Thevenin voltage experienced at a node $i$ is obtained from a reduced network as the ratio between open-circuit voltages at node $i$ and $j$ when a unit current is injected in node $j$. These open-circuit voltages may be obtained from solving the inverse problem of (6). Hereafter the coefficient may be obtained from (7).

$$
\begin{gathered}
{\left[\begin{array}{c|cc}
\mathbf{Y}_{\mathbf{n c}} & Y_{l i n k,(\bullet, i)} & Y_{l i n k,(\bullet, j)} \\
\hline Y_{l i n k,(i, \bullet)} & Y_{i, i} & Y_{i, j} \\
Y_{l i n k,(j, \bullet)} & Y_{j, i} & Y_{j, j}
\end{array}\right]\left[\begin{array}{c}
\vdots \\
\hline v_{i} \\
v_{j}
\end{array}\right]=\left[\begin{array}{c}
\mathbf{0} \\
\hline 0 \\
1
\end{array}\right]} \\
G T C_{(i, j)}=\frac{v_{\left.i\right|_{I_{j}=1} ^{O C}}}{v_{\left.j\right|_{I_{j}=1} ^{O C}}}
\end{gathered}
$$




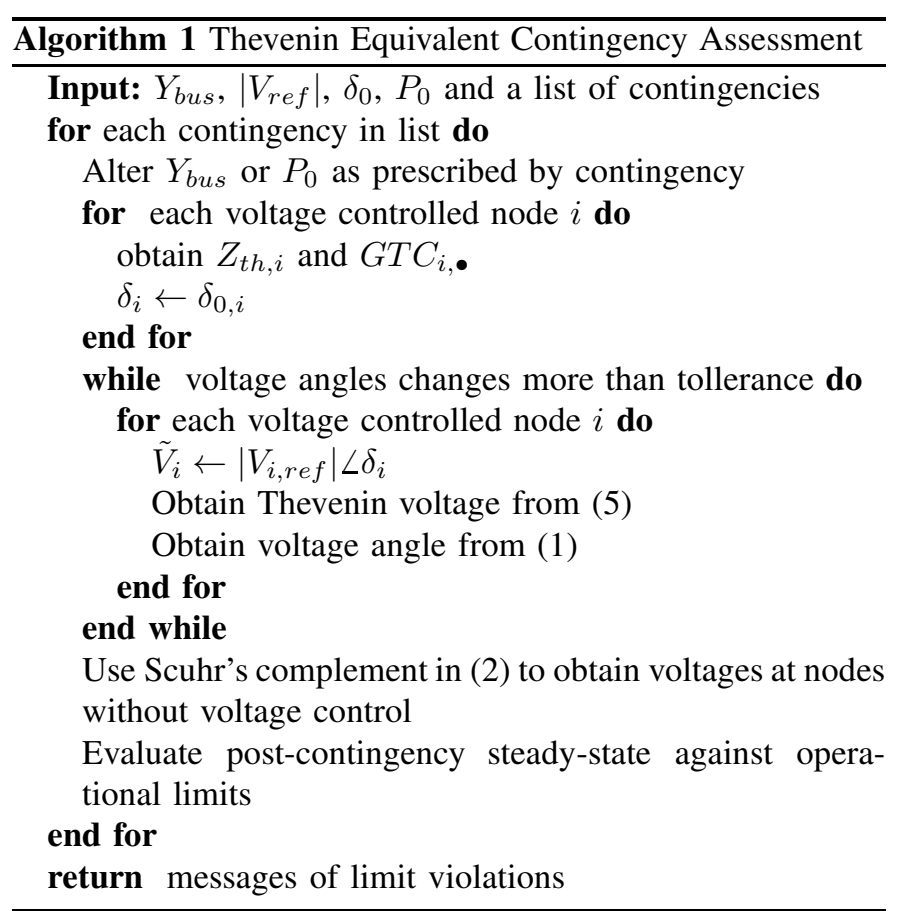

\section{B. Assessment Algorithm}

The method of obtaining post-contingency nodal voltages on basis of Thevenin equivalent representation was demonstrated in [1] where solutions for post-contingency steady-states were found to be in good agreement with time-domain simulations. The procedure is stated in algorithm 1 for quick reference.

\section{Aperiodic Small Signal Stability}

Small signal stability is a term used to describe the ability of power systems to sustain stable operation when subjected to small disturbances. A loss-of-line contingency cannot be regarded a small disturbance. But it is of interest to the operator to know if the system will be vulnerable to small disturbances in a post-contingency steady-state. The term small signal stability covers two different mechanisms in rotor angle stability [15]:

- Oscillatory small signal stability describes electromechanical oscillations due to insufficient damping torque.

- Aperiodic small signal stability describes the phenomena of synchronous machines gradually drifting out of synchronism due to insufficient synchronizing torque.

In the following attention will be focused on aperiodic small-signal rotor angle stability (ASSRAS). The benefit of the Thevenin equivalent method for solving the post contingency steady-state is that it may be realized directly from the problem that the system is post-contingency ASSRAS unstable. Insufficient synchronizing torque means that the mechanical torque on a generator's shaft exceeds the amount of electro-magnetic torque that can be presented by the loading of the generator. Considering the synchronizing torque component which varies in phase with rotor angle deviations the phenomena can be identified as a saddle-node bifurcation in figure 2 for a contingency which would cause the stable and the unstable equilibria of the $P-\delta$ curve to meet and disappear [16].

The TESCA method for contingency assessment finds the equilibria of the injected power and the $P-\delta$ curve. If no equilibria exist the inverse cosine of (1) will not be defined for the case in question. This is a criteria that reflects the ASSRAS stability limit:

$$
\left|\frac{P_{m e c, i}\left|Z_{t h, i}\right|^{2}-R_{t h, i}\left|V_{i}\right|^{2}}{\left|Z_{t h, i}\right| \cdot\left|V_{i}\right| \cdot\left|E_{t h, i}\right|}\right|\left\{\begin{array}{l}
>1 \Rightarrow \text { unstable } \\
=1 \Rightarrow \text { on margin } \\
<1 \Rightarrow \text { ASSRAS stable }
\end{array}\right.
$$

The post-contingency ASSRAS criteria in (8) can be evaluated after obtaining the Thevenin voltages in the whileloop in algorithm 1. The ASSRAS unstable cases will not converge to a post-contingency steady-state but the instability will be detected by the evaluation of (8). For the cases that do converge the distance to instability of generators can be assessed and visualized with the method given in [7] and [6].

\section{NUMERIC TEST}

A numeric test has been conducted by applying TESCA with screening for ASSRAS on a test system. The contingencies found to cause instability were afterwards evaluated using time domain simulations. The proof of concept has been limited to loss-of-line contingencies.

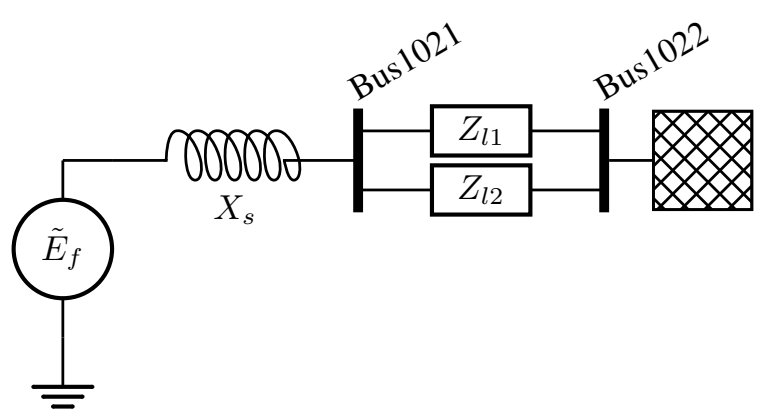

Fig. 3. Modifications to the Nordic 32 test system

The test system used was a modification of the Nordic 32 Cigré test system. The system was modified to make it prone to ASSRAS instability by removing a generating unit from bus 1022 and changing the exciter of the $200 \mathrm{MW}$ unit at bus 1021 to manually excited. In TESCA the manually excited machine was modelled as an internal voltage $\tilde{E}_{f}$ of constant magnitude behind the synchronous reactance.

TESCA was used to identify contingencies causing aperiodic small signal instability. Two different cases of loss-of-line contingencies were found to cause this type of instability for the generator at bus 1021 . The first case identified by TESCA to cause ASSRAS instability was loss of either of the lines connecting bus 1021 and 1022. The time response of this event was simulated using PSS/E. Results for voltage angles and the rotor angle for the unstable machine are plotted in figure 4 . The second contingency causing ASSRAS instability was tripping of the line connecting buses 4011 and 4021. Time response of 
TABLE I

CONTINGENCIES FOUND TO CAUSE ASSRAS INSTABILITY

\begin{tabular}{l|c} 
Detected case of ASSRAS instability & ASSRAS unstable machine \\
\hline \hline loss of line $1021-1022$ circuit 1 & unit $1021: 1$ \\
loss of line $1021-1022$ circuit 2 & unit $1021: 1$ \\
loss of line $4011-4021$ & unit $1021: 1$
\end{tabular}

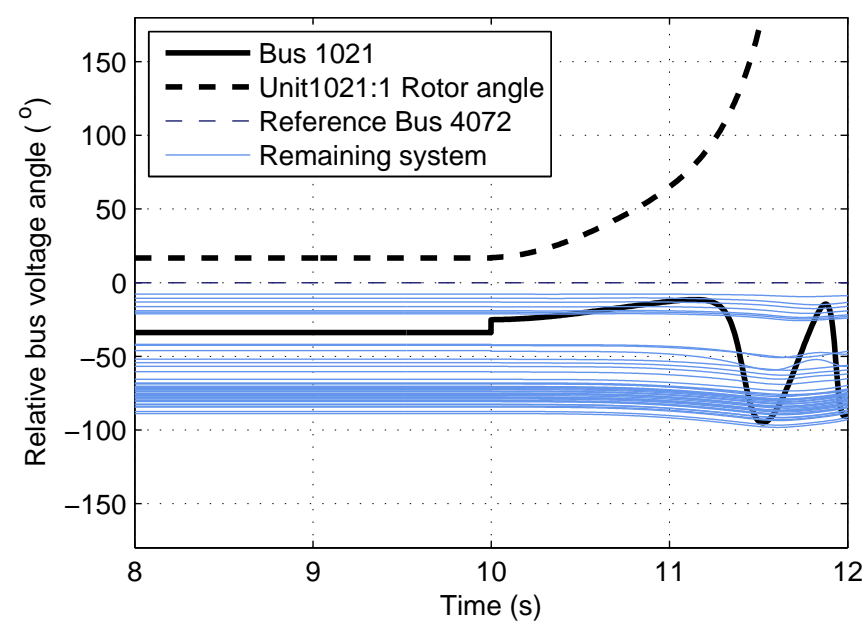

Fig. 4. Development in voltage angles following tripping of line between bus 1021 and bus 1022 at $t=10 \mathrm{~s}$

this event is found in figure 5. TESCA identified unit 1021:1 as the unstable machine in both scenarios. The time-domain simulations proved this result to be incorrect for the last case. However, the method correctly detected the that the cases are ASSRAS unstable.

As seen in figure 4 tripping one of the lines connecting the generator at bus 1021 with the remaining system causes the machine to drift out of synchronism. This incidence is a case of ASSRAS instability related to a single machine.

On figure 5 tripping the line connecting buses 4011 and 4021 causes groups of generators to drift apart and eventually a split-up of the system. This again is a case of insufficient synchronizing torque and aperiodic small signal instability. However, this case is inherently different from the one in figure 4 because it relates to the response of multiple of machines.

\section{DisCUSSION OF RESULTS}

Both cases of post-contingency aperiodic small signal instability were detected as a bifurcation point by TESCA. The method will therefore improve the situational awareness of operators. The information will be particularly useful together with means of assessing distance to instability for all the converging post-contingency steady-states.

TESCA detected the generator at bus 1021 as the unstable machine in both cases. This was true for the single-machine case. The multi-machine case caused groups of generators to drift apart and this performance was not detected by TESCA.

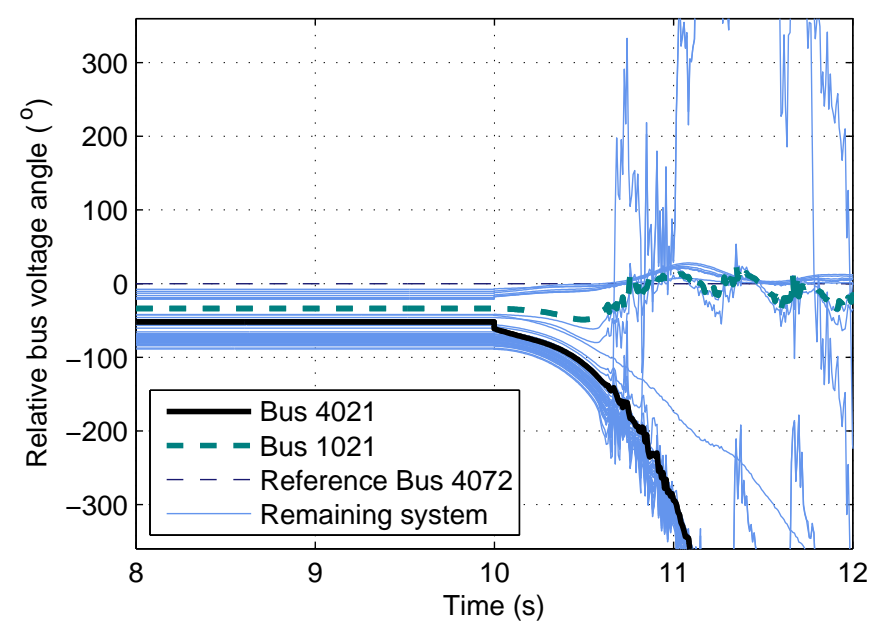

Fig. 5. Development in voltage angles following tripping of line between bus 4011 and bus 4021 at $t=10 \mathrm{~s}$

A mean of distinction between single-machine and multimachine instability must be formulated separately to allow proper remedial actions to be allocated.

The system representation applied with this proof of concept should undergo further revision to resemble the physical properties of power systems closer. The constant impedance loads is a simplification and methods for integrating composite and dynamic load models deserve attention in future work. Incorporating over- and under-excitation limiters in generator models will improve credibility of the stability assessment which rely on constant voltage magnitudes at voltage controlled nodes.

\section{CONCLUSION}

It is argued that integrating contingency assessment with methods for assessing distance to instability requires a convergent post-contingency steady-state. For contingencies which render a system unstable the distance to instability can thus not be assessed. A Thevenin equivalent method for contingency assessment was integrated with screening for bifurcation points caused by aperiodic small-signal instability. The assessment method was evaluated by conducting contingency assessment on a test system where it proved capable of detecting the unstable cases. It is expected that such information may improve situational awareness when applied in combination with means of assessing distance to instability for remaining set of converging post-contingency steady-states.

\section{REFERENCES}

[1] J. Møller, H. Jóhannsson, and J. Østergaard, "Computation of steady state nodal voltages for fast security assessment in power systems," Proceedings of Power Quality and Reliability Conference, (PQ), 2014.

[2] J. S. Thorp and S. A. Naqavi, "Load flow fractals," Proceedings of the 28th conference on Decision and Control, December 1989.

[3] J. S. Thorp, S. A. Naqavi, and H. D. Chiang, "More on load flow fractals," Proceedings of the 29th conference on Decision and Control, December 1990. 
[4] Y. V. Makarov, P. Du, S. Lu, T. B. Nguyen, X. Guo, J. W. Burns, J. F. Gronquist, and M. A. Pai, "Pmu-based wide-area security assessment: Concept, method and implementation," Ieee Transactions On Smart Grid, vol. 3, September 2012.

[5] R. Dunlop, R. Gutman, and P. Marchenko, "Analytical development of loadability characteristics for ehv and uhv transmission lines," IEEE Transactions on Power Apparatus and Systems, March/April 1979.

[6] H. Jóhannsson, Development of Early warning methods for electric power systems. $\mathrm{PhD}$ thesis, Technical University of Denmark, 2011.

[7] H. Jóhannsson, J. Østergaard, and A. H. Nielsen, "Identification of critical transmission limits in injection impedance plane," International Journal of Electrical Power \& Energy Systems, vol. 43, no. 1, p. 433443, 2012.

[8] G. Y. Yang, H. Jóhannsson, M. Lind, R. Garcia-Valle, M. Blanke, A. H. Nielsen, and J. Østergaard, "Addressing the security of a future sustainable power system: The danish sospo project," Proceedings of the 9th IET International Conference on Advances in Power system Control, 2012.

[9] J. T. G. Weckesser, H. Jóhannsson, J. Østergaard, and T. V. Cutsem, "Investigation of transient stability assessment methods to real-time operation," Proceedings of the 18th Power Systems Computation Conference (PSCC), 2014

[10] A. Perez, H. Jóhannsson, P. Vancraeyveld, and J. Østergaard, "Suitability of voltage stability study methods for real-time assessment," Proceedings of the 4th IEEE PES Innovative Smart Grid Technologies (ISGT) European Conference, 2013.

[11] A. Perez, H. Jóhannsson, and J. Østergaard, "Evaluation of enhancements to thevenin equivalent based methods for real-time voltage stability assessments," Proceedings of the 5th IEEE PES Innovative Smart Grid Technologies (ISGT) European Conference, 2014.

[12] J. T. G. Weckesser, H. Jóhannsson, J. Østergaard, and T. V. Cutsem, "Sensitivity based assessment of transient voltage sags caused by rotor swings," Proceedings of the 18th Power Systems Comp2ndutation Conference (PSCC), 2014.

[13] M. L. Wittrock, H. Jóhannsson, and A. H. Nielsen, "Wide area prosumtotion control and sensitivity pf aperiodic smal signal stability indicators," Proceedings of the 5th IEEE PES Innovative Smart Grid Technologies (ISGT) European Conference, 2014.

[14] E. Dmitrova, H. Jóhannsson, and A. H. Nielsen, "Assessment of the impact that individual voltage source has on a generator's stability," Proceedings of 10th International Power and Energy Conference, (IPEC), 2012

[15] P. Kundur, Power System Stability and Control. McGraw-Hill, 2nd ed., 1993.

[16] V. Venkatasubramanian, H. Schattler, and J. Zaborszky, "Bifurcations and feasibility regions in differential-algebraic systems," IEEE Transactions On Automatic Control, vol. 40, 1995. 